RUNNING HEAD: AN INVESTIGATION OF THE DIVERGENCES AND CONVERGENCES

OF TRAIT EMPATHY ACROSS TWO CULTURES

\title{
An Investigation of the Divergences and Convergences of Trait Empathy across Two Cultures
}

Parvaneh Yaghoubi Jami ${ }^{\mathrm{a}^{*}}$, Behzad Mansouri ${ }^{\mathrm{a}}$, Stephen J Thoma ${ }^{\mathrm{a}}$, Hyemin Han $^{\mathrm{a}}$

${ }^{a}$ University of Alabama, Tuscaloosa, USA

Author Note:

Parvaneh Yaghoubi Jami, Department of Educational Studies, University of Alabama, Tuscaloosa, AL, Box 870231, (205) 887-3508,pyaghoubijami@crimson.ua.edu

Behzad Mansouri, Department of Curriculum and Instruction, University of Alabama, Tuscaloosa, AL, Box 870232, (205) 886-9418, behman@gmail.com

Stephen J Thoma, Department of Educational Studies, University of Alabama, Tuscaloosa, AL, Box 870231, (205) 348-8146, sthoma@,ua.edu

Hyemin Han, Department of Educational Studies, University of Alabama, Tuscaloosa, AL, Box 870231, (205) 348-0746, hyemin.han@ua.edu

Corresponding concerning this article should be addressed to Parvaneh Yaghoubi Jami, Department of Educational Studies, University of Alabama, Tuscaloosa, AL, Box 870231, (205) 887-3508, pyaghoubijami@crimson.ua.edu 


\section{Authors Biographical Note:}

Parvaneh Yaghoubi Jmai is a doctoral student in Educational Psychology and Educational Neuroscience at the University of Alabama. Her research interests include cultural differences in empathic behavior, personality and social development in adolescence, social neuroscience, and personality assessment. She is currently working on neural correlates of people's empathetic responses in social settings and evaluating the psychometric properties of psychological questionnaires.

Behzad Mansouri is a doctoral student in Curriculum and Instruction at the University of Alabama. His research interests include socio-cultural basis of behavior, socio-cultural foundation of identity and agency, psycholinguistics, and cognitive neuroscience. Stephen J Thoma is a Professor in Educational Psychology at the University of Alabama. His research interests include moral judgment development, moral education, and personality and social development in late adolescence and youth.

Hyemin Han is an Assistant Professor in Educational Psychology and Educational Neuroscience at the University of Alabama. His research interests include moral development, moral education, social neuroscience, and educational interventions. 


\begin{abstract}
The extent to which individuals with a variety of cultural backgrounds differ in empathic responsiveness is unknown. This paper describes the differences in trait empathy in one independent and one interdependent society (i.e., United States and Iran respectively). The analysis of data collected from self-reported questionnaires answered by 326 adults indicated a significant difference in the cognitive component of empathy concerning participants' affiliation to either egocentric or socio-centric society: Iranian participants with interdependent cultural norms, reported higher cognitive empathy compared to American participants who share independent cultural norms. In line with previous studies, gender differences were observed in all subscales of questionnaires except the Empathy Quotient. Female participants demonstrated more empathy than males in both samples. Implications for understanding the cross-cultural differences of various components of empathy are discussed.
\end{abstract}

Keywords: Cultural differences, cognitive empathy, affective empathy, personality trait 
An Investigation of the Divergences and Convergences of Trait Empathy across Two Cultures

Morality and empathy are regarded as the most vital components of human social interaction and emotional life (Decety \& Cowell, 2014). Eisenberg-Berg and Mussen (1978) and Westman and Lewandowski (1991) demonstrated a significant association among empathy, moral reasoning, and moral behavior. Accordingly, empathetic people would develop higher levels of moral reasoning in the form of Kohlberg's post-conventional moral reasoning. Moreover, it is suggested that empathizing with others' distress and pain triggers people's moral principles motivating them to resist moral violations and transgressions (Eisenberg \& Morris, 2001), and injustices and victimization in society (Gibbs, 1991).

Following Davis (1983) empathy has been mainly investigated from a componential perspective named as affective and cognitive. Affective empathy refers to sharing similar emotional states between observer and stimulus person as a prerequisite of empathic feelings toward the target of empathy (Cuff, Brown, Taylor, \& Howat, 2016). Two subcomponents have been provisioned for affective empathy, namely as personal distress and empathic concern, each resulting in different emotional outcomes in an observer. The former happens when the observer cannot distinguish between her/his feelings and the distressed individual's (Endresen \& Olweus, 2001). The latter is a feeling of concern that observer has for another individual that will shift the focus from one's own feeling toward the other's feelings (Eisenburg \& Miller, 1987).

On the other hand, cognitive empathy is associated with perspective taking, and requires understanding another individual's thoughts and feelings and a conscious differentiation between one's own emotional state and that of others (Decety \& Michalska, 2010; Schieman \& Van 
Gundy, 2000). Previous research has established that there is a significant and positive correlation between perspective taking and moral and prosocial reasoning (Carlo, Eisenberg, \& Knight, 1992; Eisenberg, Zhou, \& Koller, 2001). For instance, Walker's (1980) longitudinal study showed that the development of perspective taking is a required condition for the development of moral reasoning. Hence, it would be necessary to have mature perspective taking abilities to make more sophisticated and advanced moral judgments as Kohlberg (1984) and Piaget (1948) hypothesized and argued in their theories on moral development.

\section{Empathy and Culture}

Generally, culture is defined as "shared knowledge and mutual expectations produced, disseminated, and reproduced among a network of interactive individuals" (Grossmann \& Na, 2014, p.2). In cultural studies Hofstede's model (Hofstede, Hofstede, \& Minkov, 2010) and Project Globe (House, Javidan, \& Dorfman, 2001) have been extensively used as theoretical frameworks for studying this phenomenon. The former model argues for culture as a multidimensional concept consisting of six major dimensions: Power distance, uncertainty avoidance, individualism/collectivism, masculinity/femininity, long-term/short-term orientation, and indulgence/restraint. House et al. (2001) expanded Hofstede's model by adding more dimensions to cultural differences. Accordingly, culture can be categorized into nine dimensions namely, uncertainty avoidance, power distance, societal collectivism, in-group collectivism, gender egalitarianism, assertiveness, future orientation, performance orientation, and humane orientation.

Empathy like other individual personality characteristics is most often influenced by the cultural norms people adopt (Cheon et al., 2011; Chopik, O’Brien, \& Konrath, 2017). Recently, 
Cross-Cultural Approach to Empathy

Chopik and collaborators' (2017) large-scale study showed that participants from collectivist cultures scored higher on self-reported empathic concern (i.e., one of the aspects of affective empathy) and overall empathy. Likewise, Heinke and Louis (2009) demonstrated that higher levels of collectivism contribute to higher perspective taking and empathic concern for others.

Such cross-cultural differences in empathy also affect the relationship between empathy and moral functioning. Given the interconnected neural pathways associated with empathy (de Waal, 2008), the ability to empathize with others is shared across various cultures. However, different cultures make distinctions among which types of beings (e.g., human beings, animals, non-human artifacts) should be the object of one's empathy and to what degree moral decisions are made affecting those beings (Decety \& Cowell, 2014). Moreover, different ways of caregiving and moral education in different cultures also significantly influence the way cognitive and affective empathy is employed and what moral decisions and behaviors are initiated based on the implementation of empathetic processes (Bedford \& Hwang, 2003).

\section{Purpose of Study}

Although many research traditions explore within-culture conditions that influence the expression of empathy, cross-cultural studies of self-reported empathy are limited to few with contradictory results (Chopik et al., 2017). Some cross-cultural studies have demonstrated that collectivism was positively associated with sympathy (Dalsky, Gohm, Noguchi, \& Shiomura, 2008) and empathy in general (Chopik et al., 2017). These researchers concluded that living in a collectivist society accounts for a higher concern for others because these cultures firmly believe that one's well-being depends on the well-being of others. In contrast, Cassels, Chan, and Chung (2010) found higher trait empathy in culturally individualist participants compared to their 
culturally collectivist peers. They attributed lower level of empathy in Eastern participants to high degree of their emotional dependency on other members of the same culture. Such culturally determined emotional bonds would hinder them to employ the necessary psychological process to distinguish between their own emotion and others.

The existing discrepancy in such studies could be due to using different measures, inconsistency in operational definitions of empathy, and not distinguishing between affective and cognitive empathy. For instance, Chopik and collaborators (2017) defined empathy as "the tendency to be psychologically in tune with others' feelings and perspectives" (p. 23) and measured emotional and cognitive empathy separately using Interpersonal Reactivity Index (IRI; Davis, 1983). Their findings are prone to criticism in several aspects including (a) failing to measure personal distress, (b) using only the English version of IRI regardless of participants' linguistic background, (c) overlooking participants' degree and level of cultural integration in the specific settings, and (d) focusing on correlational instead of causal relationships among variables.

Similarly, Cassels et al.'s (2010) empathy conceptualization is more affective in nature referring to it as "ability to perceive what other people are feeling and appropriately share that emotional state" (p. 309). Consequently, their focus is on the connection between culture and affective empathy (i.e., personal distress and empathic concern scales in IRI), while failing to consider cognitive empathy and its association with culture. On the other hand, Dalsky and colleagues (2008) equated sympathy with empathy and utilized the sympathy scale (Uchida \& Kitayama, 2001) to argue about empathy without providing a clear definition for either of them.

To address the aforementioned shortcomings, the current study measured empathy using 
Cross-Cultural Approach to Empathy

Empathy Quotient (EQ; Baron-Cohen \& Wheelwright, 2004) and the IRI (Davis, 1983) to explore differences in measurement and levels of empathy (if any) when it is measured as unidimensional (the EQ) versus multidimensional (the IRI) construct. Given the multidimensional nature of empathy as discussed earlier, we expect to find more accurate result when an adequate questionnaire is employed. An additional impetus for the present study is that there are few available studies investigated trait empathy as a peripheral phenomenon in this context, which have their own limitations. For instance, Ghorbani, Watson, Hamzavy, and Weathington, (2010) failed to report participants' mean empathy scores making it difficult to compare trait empathy cross-culturally. Moreover, the reported reliability estimates were low suggesting some potential weaknesses in the measurement scale.

What remains unaddressed in the literature is how trait empathy in Iranians born and raised in a collectivist society is similar/different from their peers raised with individualistic cultural norms like the U.S. Such a study can be important because people living in these two different socio-cultural settings may perceive their feelings in relation to others differently (Javidan, \& Dastmalchian, 2003). Moreover, the often-noted gender difference in trait empathy is still unobserved within an Iranian context. Similar to previous studies conducted in Iranian context (Kharkhurin, \& Motalleebi, 2008; Razzaghi, Ramirez, \& Zehner, 2009), in the current study, we envisaged Iran and the U.S. as culturally collectivist and individualist societies based on the reported scores in the Hofstede's model. Accordingly. Iranians scored higher on power distance uncertainty avoidance, and indulgence dimensions, while, Americans had higher scores on the remaining categories (Hofstede et al., 2010). The following research questions guided our analyses: 
1. Do Americans and Iranians score similarly as their peers with comparable cultural norms (i.e., French and Chinese; Berthoz, Wessa, Kedia, Wicker, \& Grèzes, 2008; Zhao et al., 2017)?

We chose these samples because of similarity in cultural background, using the same questionnaires for measuring trait empathy, and having acceptable reliability and validity in their measurements. We hypothesized that participants with analogous cultural backgrounds would score similarly.

2. Do the components of empathy (affective and cognitive) have notable differences in their relationship with culture?

3. We anticipate that depending on the society that people live in (i.e., independent versus interdependent), different components of empathy might be more valued and prioritized. More specifically, lower personal distress and higher empathic concern (affective empathy) are expected in Americans because of their independent culture and ability in detecting source of emotion and emotion regulation. Conversely, significant role of familial and societal perspectives, group dependency, and emotional bonds would cause higher cognitive empathy for Iranians as well as higher personal distress. What is the association between participants' gender and dispositional empathy?

We expect to replicate empirical evidence in the literature that suggested higher empathy in women.

4. In what way do gender differences in trait empathy connect to participants' cultural background?

Given the hypotheses about cultural and gender differences, it is anticipated that Iranian female participants would report higher cognitive empathy, whereas American female 


\section{Participants}

Sample 1 consists of 179 college students (30 males; $M=19.36, S D=1.46$; age-range = 18-29), from a large southern university in the U.S. participating in exchange for course credit. All students were provided with online consent forms. The majority of participants ethnically reported as White Americans/ Caucasians (\%67). To keep the sample homogenous, participants with nationalities other than Iranian and American were excluded $(N=13)$. Sample 2 included 203 Iranians (38 males; $M=22.68, S D=3.90$; age-range $=17-30$ ) living either in Iran or outside the country who voluntarily consented for participation. Forty-three participants living outside Iran were excluded from analysis due to exposure to diverse cultures that might affect trait empathy (Cassels et al., 2010). Looking at empathy from a developmental perspective (Philips, MacLean, \& Allen, 2002), we used Propensity Score Matching (PSM) technique (Schumacker, 2015) to match Iranian participants with Americans based on their age, education, marital status, and gender (Table 1). 


\begin{tabular}{|c|c|c|c|c|}
\hline & \multicolumn{2}{|c|}{$\begin{array}{l}\text { American } \\
(n=166)\end{array}$} & \multicolumn{2}{|c|}{$\begin{array}{c}\text { Iranian } \\
(n=160)\end{array}$} \\
\hline \multirow{2}{*}{ Gender } & Female & Male & Female & Male \\
\hline & $86.7 \%$ & $13.3 \%$ & $84.37 \%$ & $15.63 \%$ \\
\hline Age & $19.27 \pm 1.49$ & $19.91 \pm 1.07$ & $22.82 \pm 4.15$ & $21.88 \pm 1.94$ \\
\hline \multirow{2}{*}{ Education } & Undergraduate & Undergraduate & Undergraduate & Undergraduate \\
\hline & $100 \%$ & $100 \%$ & $100 \%$ & $100 \%$ \\
\hline \multirow{2}{*}{$\begin{array}{l}\text { Marital } \\
\text { status }\end{array}$} & Single & Single & Single & Single \\
\hline & $100 \%$ & $100 \%$ & $82.2 \%$ & $100 \%$ \\
\hline \multirow{2}{*}{ Child } & No & No & No & No \\
\hline & $99.3 \%$ & $100 \%$ & $100 \%$ & $100 \%$ \\
\hline
\end{tabular}

Table 1. Socio-demographic Characteristics of American and Iranian Respondents

Note. All participants were in the same age range, single, and either completed a bachelor degree or were studying in an undergraduate level.

\section{Measures}

English and Farsi versions (for American and Iranian participants respectively) of the IRI (Davis, 1983) and the EQ (Baron-Cohen \& Wheelwright, 2004) were used in this study. Following the guidelines for translating self-report measures used in cross-cultural studies (Beaton, Bombardier, Guillemin, \& Ferraz, 2000), three expert translators translated the EQ to Farsi. The translated versions were compared and combined into one coherent version. A second group of three translators back-translated the Farsi version to English to assure survey consistency of translation procedure. Any concerns regarding language, meaning, and compatibility between the two versions were addressed through extensive group discussions. For the IRI, we adapted the Farsi version used in Ghorbani et al.'s (2010) study. 
Cross-Cultural Approach to Empathy

IRI is a multidimensional measure (Chlopan, McCain, Carbonell, \& Hagen, 1985), with 28 items in four subscales: Fantasy scale (FS), empathic concern (EC), perspective taking (PT), and personal distress (PD) that are rated on a 5-point Likert scale. Following Davis (1983), both normal and reverse coding were applied in scoring participants' responses to the items. The final score range of 0 to 28 on each scale, where a higher score specifies a higher tendency on that subscale. In its original form, FS and PT scales were proposed to measure cognitive empathy, whereas EC and PD items were designed to tap affective empathy. However, FS items' validity in measuring cognitive empathy was questioned as they might assess respondents' imagination rather than their empathic ability (for further discussion, see Baron-Cohen \& Wheelwright, 2004). As mentioned earlier, PD could not be included in measuring affective empathy due to confusion made by distress feeling in respondents' mind that will result in non-altruistic behavior (Endresen \& Olweus, 2001). Therefore, we measured affective empathy only by items of EC and cognitive empathy by statements in PT subscales. Due to its acceptable internal consistency, the IRI has been extensively used with non-English samples such as French (Berthoz et al., 2008). See Table 2 for internal consistency of the questionnaires.

On the other hand, the EQ (accessed from https://www.autismresearchcentre.com) consists of 60 items measuring empathy as a one-dimensional construct in adults. Participants rated the statements according to their degree of agreement or disagreement on a 4-point Likert scale. After excluding 20 filler items, the remaining items are scored based on the guidelines provided by Baron-Cohen and Wheelwright (2004). Accordingly, the final score will be between 0 and 80, where a higher score indicates higher empathic tendencies. The EQ's psychometric properties have been attested in numerous studies (e.g., Berthoz et al., 2008; Preti et al., 2011). Originally, the EQ was developed for clinical purposes and has a cut-off score (lower than 30) 
Cross-Cultural Approach to Empathy

for identifying possible autistic spectrum disorder (ASD). Therefore, we used the EQ to exclude participants with score lower than 30 as a potential sign of lacking empathy. It should be noted that lack of empathy in ASD population has been utterly debated (e.g., Rueda, FernándezBerrocal, \& Baron-Cohen, 2015) mainly originated from dimensionality difference in measures. For example, studies using the EQ have reported lower empathy in ASD population (BaronCohen \& Wheelwright, 2004; Berthoz et al., 2008), whereas studies using the IRI have found difference between ASD and ordinary populations only in two subscales. More specifically, ASD populations might have lower and higher scores in cognitive empathy and personal distress respectively, but they could score similarly in affective empathy (Rueda et al., 2015; Senland \& Higgins-D'Alessandro, 2016). The higher personal distress might impede empathic concern feelings in people with ASD and prevent them from showing empathic behavior.

Table 2. Reliability Validation of English and Farsi versions of Questionnaires

Farsi Version

IRI-PD

IRI-EC

IRI-PT

15-item EQ
.69

.68

.63

.84

\section{English Version}

.69

.79

.75

.82

Construct Equivalence of Measurements. Principal Components Analysis (PCA) was conducted on responses to both English and Farsi versions of the EQ for evaluating measurements' similarity. Using these results, 15 items with loadings greater than 0.30 on the first (principal) component (Raykov \& Marcoulides, 2011) were identified in both samples. The 15 -item subset in both samples showed statistically significant model-fit: English version, $\chi^{2}$ (76) 
Cross-Cultural Approach to Empathy

$=112.810, p=.004$; and Farsi version, $\chi^{2}(76)=229.116, p<.001$. The short versions of the EQ used for both samples consist of the same items with a final score variation between 0 and 30 . Appendix A provides details about the included items and final factor loadings for these items in both samples.

\section{Procedure}

American participants were recruited from students enrolled in university classes. All interested participants received the online link for the surveys used in this study. The link directed each participant to consent form, demographic questionnaire, and two empathy surveys. For recruiting potential Iranian participants, a flyer explaining the study was posted through social media (e.g., Facebook, Instagram) to be visible in Farsi speakers' news feed. The translated versions of questionnaires and consent form were sent to interested participants through a Qualtrics link. For the sake of similarity between the two samples the order of questionnaires was the same.

\section{Data Analyses}

To answer the first research question, one-sample t-tests were conducted. For this analysis, we compared our sample' scores of the IRI subscales (i.e., EC, PD, and PT) with the means and standard deviations of the same subscales reported in previous studies of French and Chinese participants. The reported internal consistency used with Chinese sample was acceptable, $\boldsymbol{\alpha}_{\text {IRI }}=.66-.79$ (Zhao et al., 2017). Berthoz et al. (2008) did not examine the reliability of the IRI used with their sample. Instead, the authors relied on the previously reported reliability index for the IRI and concluded, "The IRI has good internal and convergent validity and test-retest reliability" (p. 471). To answer the remaining research questions (evaluating the 
possible effect of culture, gender, and their interaction) multivariate analysis of variance (MANOVA) was conducted. Specifically, a 2 x 2 MANOVA was applied in which nationality (i.e., Iranian and American) and gender were independent and participants' scores on IRI_EC, IRI-PD, IRI-PT, and 15-item EQ were dependent variables. Moreover, Type III Sum Square in MANOVA was used for unbalanced gender representation in the sample (Pituch \& Stevens, 2015). Participants' scores on IRI-FS were excluded due to ongoing debate about its validity in measuring empathy (Baron-Cohen \& Wheelwright, 2004). All analyses were conducted using IBM SPSS statistic 24 .

\section{Result}

To calculate the mean score in each scale, descriptive data analyses were conducted for each group of participants. In the IRI questionnaire answered by American participants, the lowest score was for IRI-PT, and the highest was in IRI-EC subcomponents $(M=14.80, S D=$ 4.76; and $M=20.73, S D=4.18$ respectively). Similarly, French participants used in Berthoz et al.'s (2008) study, scored higher on IRI-EC $(M=19.7, S D=4.5)$ compared to their scores on IRI-PT $(M=17, S D=3.8)$. The result of one-sample t-test showed that on average American participants scored significantly lower than French participants on IRI-PT, $t(165)=-5.974, p<$ $.001, d=.51$. On the other hand, on the IRI-EC and IRI-PD subscales, Americans in the current study scored significantly higher than the mentioned French population; IRI-EC, $t(165)=3.193$, $p=.002, d=.24 ;$ IRI-PD, $t(165)=7.191, p<.001, d=.57$.

Focusing on Iranian participants, the lowest score was for IRI-PD $(M=15.39, S D=4.41)$ and the highest was in IRI-EC $(M=19.13, S D=4.05)$ subcomponents. Iranian participants' empathic concern mean scores was slightly higher than the reported mean score of Chinese 
sample $(M=18.63, S D=3.68)$ in Zhao and colleagues' study $(2017)$, however, it was not significant, $t(159)=1.566, p=.119, d=.13$. On the contrary, Iranians' score on IRI-PD and IRI-PT were significantly different than the reported mean for Chinese sample $(M=13.59, S D=$ 4.38; $M=17.27, S D=5.54$, respectively); IRI-PD, $t(159)=5.169, p<.001, d=.41 ;$ IRI-PT, $t$ $(159)=-3.458, p=.001, d=.28$.

\section{Empathy and Culture}

The result of MANOVA suggested a significant multivariate main effect of nationality on trait empathy, Wilks' $\lambda=.879, F(4,319)=10.927, p<.001, \eta^{2}=.121$. Therefore, at least one of the subscales in the questionnaires is affected by the nationality of participants. Significant univariate main effects for nationality were found only for IRI-PT, $F(1,322)=21.851, p<.001$, $\eta^{2}=.064 ;$ and the EQ, $F(1,322)=10.983, p=.001, \eta^{2}=.033$. The result did not reach a significant level for the other subscales; IRI-EC, $F(1,322)=.003, p=.954, \eta^{2}=.000$; and IRI$\mathrm{PD}, F(1,322)=.037, p=.847, \eta^{2}=.000$. As the pairwise comparison results suggested, Iranian participant' scores on perspective taking was significantly higher than their Americans peers. On the other hand, American participants reported higher score in the EQ, which was significantly higher than Iranians. On the other two subscales both groups of participants, scored similarly. Cultural differences in each subscale are given in Table 3. 
Table 3. Comparison of Self-Reported Empathy based on Culture

\begin{tabular}{lccccc} 
& Nationality & $M$ & $S D$ & \multicolumn{2}{c}{$95 \%$ Confidence Interval } \\
\hline \multirow{2}{*}{ IRI-EC } & & & Lower Bound & Upper Bound \\
& Iranian & 19.13 & 4.05 & 18.50 & 19.76 \\
IRI-PD & Americans & 20.73 & 4.18 & 20.09 & 21.37 \\
& Iranian & 15.39 & 4.41 & 14.70 & 16.08 \\
IRI-PT & Americans & 15.15 & 4.93 & 14.40 & 15.91 \\
& Iranian & $16.23^{* *}$ & 3.82 & 15.63 & 16.82 \\
15 -item EQ & Americans & 14.80 & 4.75 & 14.07 & 15.52 \\
& Iranian & 13.56 & 4.93 & 12.79 & 14.33
\end{tabular}

Note. ${ }^{* *}$ The difference is significant at the .01 level

Table 4. Descriptive Statistic of Empathy traits in Male and Female

\begin{tabular}{lccccc}
\hline & Gender & $M$ & $S D$ & \multicolumn{2}{c}{$95 \%$ Confidence Interval } \\
\hline \multirow{2}{*}{ IRI-EC } & & & Lower Bound & Upper Bound \\
& Male & 18.26 & 4.66 & 16.89 & 19.62 \\
IRI-PD & Female & $20.23^{* *}$ & 4.04 & 19.76 & 20.71 \\
& Male & 13.79 & 4.55 & 12.45 & 15.12 \\
IRI-PT & Female & $15.52^{* *}$ & 4.66 & 14.97 & 16.07 \\
& Male & 13.91 & 5.75 & 12.23 & 15.60 \\
15 -item EQ & Female & $15.76^{* *}$ & 4.05 & 15.29 & 16.24 \\
& Male & 15.09 & 4.31 & 13.82 & 16.35 \\
& Female & 15.01 & 4.94 & 14.43 & 15.60
\end{tabular}

Note. ${ }^{* *}$ The difference is significant at the .01 level

\section{Empathy and Gender}

As expected, participants' gender orientation significantly affected their response to at least one of the scales, Wilks' $\lambda=.926, F(4,319)=6.418, p<.001, \eta^{2}=.074$. Significant univariate main effects of gender were obtained for IRI-EC, $F(1,322)=10.192, p=.002, \eta^{2}=.031$; IRI- 
Cross-Cultural Approach to Empathy

PT, $F(1,322)=9.371, p=.002, \eta^{2}=.028$; and IRI-PD, $F(1,322)=5.572, p=.019, \eta^{2}=.017$.

Women and men did not differ significantly in the EQ, $F(1,322)=.059, p=.809, \eta^{2}=.000$. On average, women had a higher score in empathic concern, perspective taking, and personal distress (Table 4).

Interaction between Nationality and Gender. Along with the hypothesis, the result of MANOVA suggested in at least one scale, participants' score significantly differ depending on their nationality and gender, Wilks' $\lambda=.943, F(4,319)=4.829, p=.001, \eta^{2}=.057$. The univariate interaction of nationality and gender was obtained for IRI-EC, $F(1,322)=12.584, p<.000, \eta^{2}=$ .038 ; and IRI-PT, $F(1,322)=11.866, p=.001, \eta^{2}=.036$. The interaction of nationality and gender was not significant in IRI-PD subscale, $F(1,322)=.074, p=.786, \eta^{2}=.000$; and the EQ, $F(1,322)=.727, p=.395, \eta^{2}=.002$.

The results of a pairwise comparison indicated that Iranian males reported slightly higher scores on all the subscales, except IRI-PD, compared to Iranian female participants; however the difference was not significant (all $p \mathrm{~s}>.05$ ). Contrarily, American females scored significantly higher than their American male peers on IRI-EC and IRI-PT $(p<.01)$, but not on IRI-PD $(p=$ .08). Focusing on each subscale, participants' scores in perspective taking had the most dependency on their nationality and gender. Accordingly, American male participants had the lowest scores compared to other participants, whereas Iranian male participants had the highest scores. Similarly, Iranian females reported slightly higher perspective taking than American females. Focusing on the other subscales, on the empathic concern, both American females and Males had the highest and lowest scores respectively compared to Iranians. On the other hand, on personal distress, Iranian males and females had the lowest and highest scores compared to 
Cross-Cultural Approach to Empathy

their American counterparts. On the last subscale, the EQ, Iranian females reported the lowest scores, whereas American females had the highest scores. See Figure 1 for details.

Figure 1. Mean scores on each questionnaire based on participants' nationality and gender. In all subscales, except personal distress, American male participants had the lowest score.

\section{Discussion}

Research has evidenced that culture, among other factors, is a correlate of empathetic behavior (Chopik et al., 2017), however, there is little consensus in the research examining empathetic perceptions among people with contrasting cultural backgrounds (i.e., collectivism versus individualism) While some studies suggested higher empathy in collectivist societies (Dalsky et al., 2010), others reported the opposite (Cassels et al., 2010). The existing inconsistencies are attributed to methodological discrepancy in measuring empathy, lack of rigorous definition, and failure to distinguish between affective and cognitive facets of empathy. To address these limitations, the current study administered multiple questionnaires to investigate perceptions of affective and cognitive empathy in samples from collectivist (Iran) and individualist (USA) cultures.

As the findings suggest, the two groups of participants are alike in affective empathy (measured by IRI-EC), but different in cognitive empathy (measured by IRI-PT). Accordingly, Iranian participants scored significantly higher in perspective taking compared to their American counterparts. Similar findings were also reported in a study comparing Chinese and American perspective taking ability (Wu \& Kaysar 2007). Given that all the other distinctions, including personal distress and empathic concern were similar between both samples and confirms a relationship between prosocial behavior and personal distress (Cassels et al., 2010), we believe that higher levels of cognitive empathy among Iranians is due to more mature perspective-taking 
Cross-Cultural Approach to Empathy

ability. Situations that trigger high personal distress typically result in "self-oriented" feelings (Batson, 2009), however, Iranians' responses on perspective taking, suggests otherwise.

Therefore, it seems logical to conclude that higher levels of cognitive empathy among Iranian participants could be traced to their cultural context. Living in collectivist and socio-centric societies may promote more behaviors related to taking others' perspective, interpreting one's actions, and empathizing for the good of increasing the welfare of others.

Cultural influence was also observed in participants' response to the EQ with Americans reporting higher levels of agreement with its statements. The EQ structure could contribute to such results as it treats empathy as a unidimensional construct. As a result, when the instrument does not differentiate between affective and cognitive empathy it is plausible that individuals with individualistic cultural norms would have higher empathy scores compared to their collectivist peers. On the contrary, when empathy is regarded as a multi-dimensional construct and its components are separately measured, the pattern of association between empathy and culture suggests otherwise. An alternative explanation may related to the translating procedure used in the current study. Some of the EQ items were more difficult for Iranians to endorse, which caused a different item ordering in the Farsi and English versions. However, both versions showed a high reliability and found to be a valid questionnaire for measuring empathy among Farsi-speakers (Authors, under review).

An additional objective of the current research was to examine differences in gender, which resulted in agreement with existing empirical research, supporting the conception that women are more empathic than men (Endresen \& Olweus, 2001; Schieman \& Van Gundy, 2000). Likewise, female participants in the current study felt more personal distress and empathic concern, and reported higher perspective taking abilities. Divergent rearing styles may 
Cross-Cultural Approach to Empathy

explain this difference; while girls are raised with a great emphasis on social skills oriented toward warmth and caring, boys are raised to control their emotions (e.g., Gilligan, 1982). However, such differences in parenting styles may be unique to Western cultures as our results along with another study with a Chinese (collectivistic) sample (Zhao et al., 2017) did not find gender differences in favor of female participants. Specifically, Iranian male and female participants reported similar level of empathy, while American males had a significantly different empathic response than their female American peers. In the same way, studies examining gender differences in moral sensitivity, a related concept to empathy (Decety, Michalska, \& Kinzler, 2011), evidenced similar score divergence across American and Turkish participants (Sigma-Mugan, Daly, Onkal, \& Kavut, 2005). Gender roles and identities are influenced by society and cultural norms, thus, empathic behaviors would vary across gender and culture (e.g., Atasoy, 2006; Phillips, 2003).

Regarding the EQ, the current study did not find any gender differences. Although, other studies (e.g., Baron-Cohen \& Wheelwright, 2004) reported that women significantly scored higher than men on the EQ, their findings should be cautiously interpreted. First, in the studies reporting gender differences the 40-item EQ was used, while studies using shortened versions (i.e., 28-item, Lawrence, Shaw, Baker, Baron-Cohen, \& David, 2004; 15-item, Muncer \& Ling, 2006) did not support previous findings. Comparably, when we administered the 40 -item version among American participants, higher scores for female participants were found. However, no gender differences were observed in the same sample using the shortened version (i.e., 15-item), which could be due to the included items. Thirteen items in this version were loaded under cognitive empathy and 1 item under social skill factors reported in previous studies (Lawrence et al., 2004). It is worth noting that there was no evidence of gender differences in these two factors 
Cross-Cultural Approach to Empathy

(see Muncer \& Ling, 2006). Second, studies including Eastern populations found no significant differences between male and female respondents (Kim \& Lee, 2010). Similarly, we did not observe any gender differences for Iranians in either 40 -item or 15 -item versions of the EQ.

Batson, Lishner, Cook, and Sawyer, (2005) suggested nurturance as a possible indicator of empathic behavior. Considering the nature of empathy, it seems plausible that empathy development is susceptible to cultural differences. In every culture people experience unique environments, are reared differently, adopt distinct moral values and norms, value their social bonds differently, and display behaviors that are deemed acceptable in their culture and society (Hofstede et al., 2010). For example, people would define their moral identity by societal or individualistic values depending on collectivist or individualist cultures in which they live (Jia \& Krettenauer, 2017). Cognitive empathy differed among participants from different cultures, quite possibly due to nurturance, whereas affective empathy may be a universal and innate ability in individuals (Iacoboni, 2009).

\section{Limitation and Future Direction}

It is plausible that some limitations may have influenced the results obtained, thus, need to be addressed in future research. The first limitation is associated with an unbalanced sample size, with more female participants than men. Although, we used the recommended analysis for unequal sample size (Type III Sum Squares; Pituch \& Stevens, 2015), the main effect of gender and the interaction effect of gender and culture on trait empathy should be interpreted cautiously. An additional limitation is related to the method used for data collection. American participants were recruited from university classes in exchange of course credit, while Iranians participated voluntarily and were informed through social media. Thus, the second sample had more geographical variation, albeit no effect of location was found. Third, our Iranian sample is 
Cross-Cultural Approach to Empathy

limited to those who had access to the internet, which could make the result ungeneralizable to the whole population. Finally, cognitive and affective empathy were measured using self-report questionnaires. As the evidence suggests, social desirability bias and threats to internal validity (especially response accuracy) are the most problematic issues with this type of measurement. Although, all the responses suspected to follow a pattern (i.e., similar answer to all items) were excluded in this study, future studies are encouraged to employ other types of measurements such as behavioral measurement.

\section{Implication}

To the best of our knowledge, the current study is the first to directly compare empathic responsiveness between Iranians and Americans as representatives of collectivist and individualist cultures. Despite mentioned limitations, we believe our work is a point of reference for future studies, both in and beyond the Iranian context. Cross-cultural research on affective and cognitive aspects of empathy can reveal how these concepts are perceived and valued across different cultures leading to a more robust understanding of human beings' social life. As our findings demonstrated, empathy is both universal and culture-bound, depending on the specific component under investigation. Accordingly, examining affective empathy across participants with collectivist and individualistic cultural norms demonstrates its universality. Conversely, living in an interdependent society would foster perspective taking ability in assigning equal weight to one's and others' emotional and cognitive state (Wu \& Keysar, 2007).

Additionally, with the increasing rate of immigration to more westernized countries and the spread of global tendency toward ego-centric values, especially in commonly-known collectivist societies (Santos, Varnum, \& Grossmann, 2017), cross-cultural studies such as the current study can contribute to understanding the underlying cultural and psychological factors 
Cross-Cultural Approach to Empathy

leading to (dis)integrations of immigrants into host cultures. As immigrants enter a new country, many desire to be immersed in their new communities by adopting similar behaviors and cultural norms as the citizens of that country. Therefore, having prior knowledge about the cognitive and affective foundation of social interactions in the different culture can facilitate a successful integration process and help individuals selectively apply aspects of new culture into their own.

Our study could also have implications for moral education. As mentioned earlier, empathy and morality share a complementary relationship (de Wall, 2009). Educators may be able to learn what kind of moral educational components in other countries positively contributes to empathy and moral development by comparing empathy scores between different cultures and countries as we did in the present study. Educators in each country may consider examining moral education in the opposite country to learn which aspects of moral education contribute to the relatively higher scores in a specific domain of empathy. Moreover, examining cultural influences on empathy development can also contribute to the development of moral educational programs that may work more effectively in a specific culture. Understanding cultural differences in empathy might provide useful insight to moral educators about how to develop culturally-relevant and attainable moral educational programs that promote empathy development. 


\section{References}

Atasoy, Y. (2006). Governing women's morality. European Journal of Cultural Studies, 9(2), 203-221. doi:10.1177/1367549406063164

Baron-Cohen, S., \& Wheelwright, S. (2004). The Empathy Quotient: An Investigation of Adults with Asperger Syndrome or High Functioning Autism, and Normal Sex Differences. Journal of Autism and Developmental Disorders, 34(2), 163-175. doi:10.1023/b:jadd.0000022607.19833.00

Batson, C. D., Lishner, D. A., Cook, J., \& Sawyer, S. (2005). Similarity and nurturance: Two possible sources of empathy for strangers. Basic and applied social psychology, 27(1), $15-25$.

Batson, C. D. (2009). These things called empathy: Eight related but distinct phenomena. In J. Decety \& W. Ickes (Eds.), Social neuroscience. The social neuroscience of empathy (pp. 3-15). Cambridge, MA, US: MIT Press.

Beaton, D. E., Bombardier, C., Guillemin, F., \& Ferraz, M. B. (2000). Guidelines for the process of cross-cultural adaptation of self-report measures. Spine, 25(24), 3186-3191.

Bedford, O., \& Hwang, K-K. (2003). Guilt and shame in Chinese Culture: A cross-cultural framework from the perspective of morality and identity. Journal for the Theory of Social Behavior, 33(2), 127-144.

Berthoz, S., Wessa, M., Kedia, G., Wicker, B., \& Grèzes, J. (2008). Cross-cultural validation of the empathy quotient in a French-speaking sample. The Canadian Journal of Psychiatry, 53(7), 469-477. 
Cross-Cultural Approach to Empathy

Carlo, G., Eisenberg, N., \& Knight, G. P. (1992). An objective measure of prosocial moral reasoning. Journal of Research on Adolescence, 2, 331-349.

Cassels, T. G., Chan, S., \& Chung, W. (2010). The Role of Culture in Affective Empathy: Cultural and Bicultural Differences. Journal of Cognition and Culture, 10(3), 309-326. doi:10.1163/156853710x531203

Cheon, B. K., Im, D. M., Harada, T., Kim, J. S., Mathur, V. A., Scimeca, J. M., ... \& Chiao, J. Y. (2011). Cultural influences on neural basis of intergroup empathy. NeuroImage, 57(2), 642-650.

Chlopan, B. E., McCain, M. L., Carbonell, J. L., \& Hagen, R. L. (1985). Empathy: Review of available measures. Journal of Personality and Social Psychology, 48(3), 635-653. doi:10.1037//0022-3514.48.3.635

Chopik, W. J., O’Brien, E., \& Konrath, S. H. (2017). Differences in Empathic Concern and Perspective Taking Across 63 Countries. Journal of Cross-Cultural Psychology, 48(1), 23-38.

Cuff, B. M., Brown, S. J., Taylor, L., \& Howat, D. J. (2016). Empathy: a review of the concept. Emotion Review, 8(2), 144-153.

Dalsky, D., Gohm, C. L., Noguchi, K., \& Shiomura, K. (2008). Mutual self-enhancement in Japan and the United States. Journal of Cross-Cultural Psychology, 39(2), 215-223.

Davis, M. H. (1983). Measuring individual differences in empathy: Evidence for a multidimensional approach. Journal of Personality and Social Psychology, 44(1), $113-$ 126. doi:10.1037//0022-3514.44.1.113

Decety, J., \& Cowell, J. M. (2014). The complex relation between morality and empathy. Trends in Cognitive Sciences, 18(7), 337-339. doi:10.1016/j.tics.2014.04.008 
Decety, J., \& Michalska, K. J. (2010). Neurodevelopmental changes in the circuits underlying empathy and sympathy from childhood to adulthood. Developmental science, 13(6), 886899.

Decety, J., Michalska, K. J., \& Kinzler, K. D. (2011). The Developmental Neuroscience of Moral Sensitivity. Emotion Review, 3(3), 305-307. doi:10.1177/1754073911402373

de Waal, F. B. M. (2008). Putting the altruism back into altruism: The evolution of empathy. The Annual Review of Psychology, 59, 279-300.

de Waal, F. (2009). The age of empathy. New York, NY: Harmony.

Eisenberg-Berg, N., \& Mussen, P. (1978). Empathy and moral development in adolescence. Developmental Psychology, 14(2), 185-186.

Eisenberg, N., \& Miller, P. A. (1987). The relation of empathy to prosocial and related behaviors. Psychological Bulletin, 101(1), 91-119. doi:10.1037//0033-2909.101.1.91

Eisenberg, N., \& Morris, A. S. (2001). The origins and social significance of empathy-related responding. A review of empathy and moral development: Implications for caring and justice by M. L. Hoffman. Social Justice Research, 14(1), 95-120.

Eisenberg, N., Zhou, Q., \& Koller, S. (2001). Brazilian adolescents’ prosocial moral judgment and behavior: Relations to sympathy, perspective taking, gender-role orientation, and demographic characteristics. Child Development, 72, 518-534.

Endresen, I. M., \& Olweus, D. (2001). Self-reported empathy in Norwegian adolescents: Sex differences, age trends, and relationship to bullying. Constructive \& destructive behavior: Implications for family, school, \& society, 147-165. doi:10.1037/10433-007 
Cross-Cultural Approach to Empathy

Ghorbani, N., Watson, P. J., Hamzavy, F., \& Weathington, B. L. (2010). Self-Knowledge and Narcissism in Iranians: Relationships with Empathy and Self-Esteem. Current Psychology, 29(2), 135-143. doi:10.1007/s12144-010-9079-5

Gibbs, J. C. (1991). Toward an integration of Kohlberg's and Hoffman's Moral Development Theories. Human Development, 34, 88-104.

Gilligan, C. (1982). In a different voice. Harvard University Press.

Grossmann, I., \& Na, J. (2014). Research in culture and psychology: past lessons and future challenges. Wiley Interdisciplinary Reviews: Cognitive Science, 5(1), 1-14.

Heinke, M. S., \& Louis, W. R. (2009). Cultural background and individualistic-collectivistic values in relation to similarity, perspective taking, and empathy. Journal of Applied Social Psychology, 39(11), 2570-2590.

Hofstede, G., Hofstede, G. J., \& Minkov, M. (2010). Cultures and organizations: Software of the mind. Revised and expanded. McGraw-Hill, New York.

House, R., Javidan, M., \& Dorfman, P. (2001). Project GLOBE: an introduction. Applied Psychology, 50(4), 489-505.

Iacoboni, M. (2009). Imitation, empathy, and mirror neurons. Annual review of psychology, 60, 653-670.

Javidan, M., \& Dastmalchian, A. (2003). Culture and leadership in Iran: The land of individual achievers, strong family ties, and powerful elite. The Academy of Management Executive, $17(4), 127-142$.

Jia, F., \& Krettenauer, T. (2017). Recognizing moral identity as a cultural construct. Frontiers in psychology, 8, 412, doi: 10.3389/fpsyg.2017.00412. 
Kharkhurin, A. V., \& Motalleebi, S. N. S (2008). The impact of culture on the creative potential of American, Russian, and Iranian college students. Creativity Research Journal, 20(4), 404-411.

Kim, J., \& Lee, S. J. (2010). Reliability and validity of the Korean version of the Empathy Quotient Scale, Psychiatry investigation, 7(1), 24-30

Kohlberg, L. (1984). The Psychology of Moral Development. San Francisco: Harper and Row, 2.

Lawrence, E. J., Shaw, P., Baker, D., Baron-Cohen, S., \& David, A. S. (2004). Measuring empathy: Reliability and validity of the Empathy Quotient, Psychological Medicine, 34(5), 911-919. doi:10.1017/s0033291703001624

Muncer, S. J., \& Ling, J. (2006). Psychometric analysis of the empathy quotient (EQ) scale. Personality and Individual differences, 40(6), 1111-1119.

Piaget, J. (1948). The moral judgment of the child (1932). New York: The Free.

Pituch, K. A., \& Stevens, J. P. (2015). Applied multivariate statistics for the social sciences: Analyses with SAS and IBM's SPSS. Routledge.

Phillips, A. (2003). When Culture Means Gender: Issues of Cultural Defense in the English Courts. Modern Law Review, 66(4), 510-531. doi:10.1111/1468-2230.6604002

Phillips, L. H., MacLean, R. D., \& Allen, R. (2002). Age and the understanding of emotions: Neuropsychological and sociocognitive perspectives. The Journals of Gerontology Series B: Psychological Sciences and Social Sciences, 57(6), P526-P530.

Preti, A., Vellante, M., Baron-Cohen, S., Zucca, G., Petretto, D. R., \& Masala, C. (2011). The Empathy Quotient: A cross-cultural comparison of the Italian version. Cognitive Neuropsychiatry, 16(1), 50-70. 
Cross-Cultural Approach to Empathy

Raykov, T., \& Marcoulides, G. A. (2011). Introduction to psychometric theory. New York, NY: Routledge.

Razzaghi, M., Ramirez, M., \& Zehner, R. (2009). Cultural patterns in product design ideas: comparisons between Australian and Iranian student concepts. Design Studies, 30(4), $438-461$.

Rueda, P., Fernández-Berrocal, P., \& Baron-Cohen, S. (2015). Dissociation between cognitive and affective empathy in youth with Asperger Syndrome. European Journal of Developmental Psychology, 12(1), 85-98.

Santos, H. C., Varnum, M. E., \& Grossmann, I. (2017). Global increases in individualism. Current Directions in Psychological Science, 28(9), 1228-1239. doi:

$10.1177 / 0956797617700622$

Senland, A. K., \& Higgins-D’Alessandro, A. (2016). Sociomoral Reasoning, Empathy, and Meeting Developmental Tasks During the Transition to Adulthood in Autism Spectrum Disorder. Journal of autism and developmental disorders, 46(9), 3090-3105.

Schieman, S., \& Van Gundy, K. (2000). The personal and social links between age and selfreported empathy. Social Psychology Quarterly, 152-174.

Schumacker, R. E. (2015). Using $R$ with multivariate statistics. SAGE Publications.

Simga-Mugan, C., Daly, B. A., Onkal, D., \& Kavut, L. (2005). The Influence of Nationality and Gender on Ethical Sensitivity: An Application of the Issue-Contingent Model. Journal of Business Ethics, 57(2), 139-159. doi:10.1007/s10551-004-4601-z

Uchida, Y., \& Kitayama, S. (2001). Development and validation of a sympathy scale. Japanese Journal of Psychology, 72(4), 275-282. 
Walker, L. J. (1980). Cognitive and perspective-taking prerequisites for moral reasoning development. Child Development, 51, 131-139

Westman, A. S., \& Lewandowski, L. M. (1991). How empathy, egocentrism, Kohlberg’s moral development, and Erikson's psychological development are related to attitudes toward war. Psychological Reports, 69, 1123-1127.

Wu, S., \& Keysar, B. (2007). The effect of culture on perspective taking. Psychological science, 18(7), 600-606.

Zhao, Q., Neumann, D. L., Cao, X., Baron-Cohen, S., Sun, X., Cao, Y., ... \& Shum, D. H. (2017). Validation of the Empathy Quotient in Mainland China. Journal of Personality Assessment, 1-10. 
Cross-Cultural Approach to Empathy

Appendix A: Results from Principal Components Analysis

\begin{tabular}{ccc}
\hline \multirow{2}{*}{ Item Number } & \multicolumn{2}{c}{ Loading on Principal Component } \\
\cline { 2 - 3 } 1 & English Speakers & Farsi Speakers \\
\hline 19 & .345 & .293 \\
22 & .586 & .447 \\
25 & .318 & .429 \\
26 & .701 & .581 \\
35 & .644 & .539 \\
36 & .288 & .331 \\
37 & .507 & .709 \\
41 & .271 & .241 \\
43 & .518 & .429 \\
44 & .406 & .705 \\
52 & .501 & .411 \\
54 & .655 & .638 \\
55 & .629 & .501 \\
58 & .627 & .529 \\
& .666 & .497 \\
Model Fit & & \\
Chi Square & 112.810 & 229.116 \\
Degrees of Freedom & 76 & 76 \\
$p$ value & $<0.05$ & $<.05$ \\
\hline
\end{tabular}




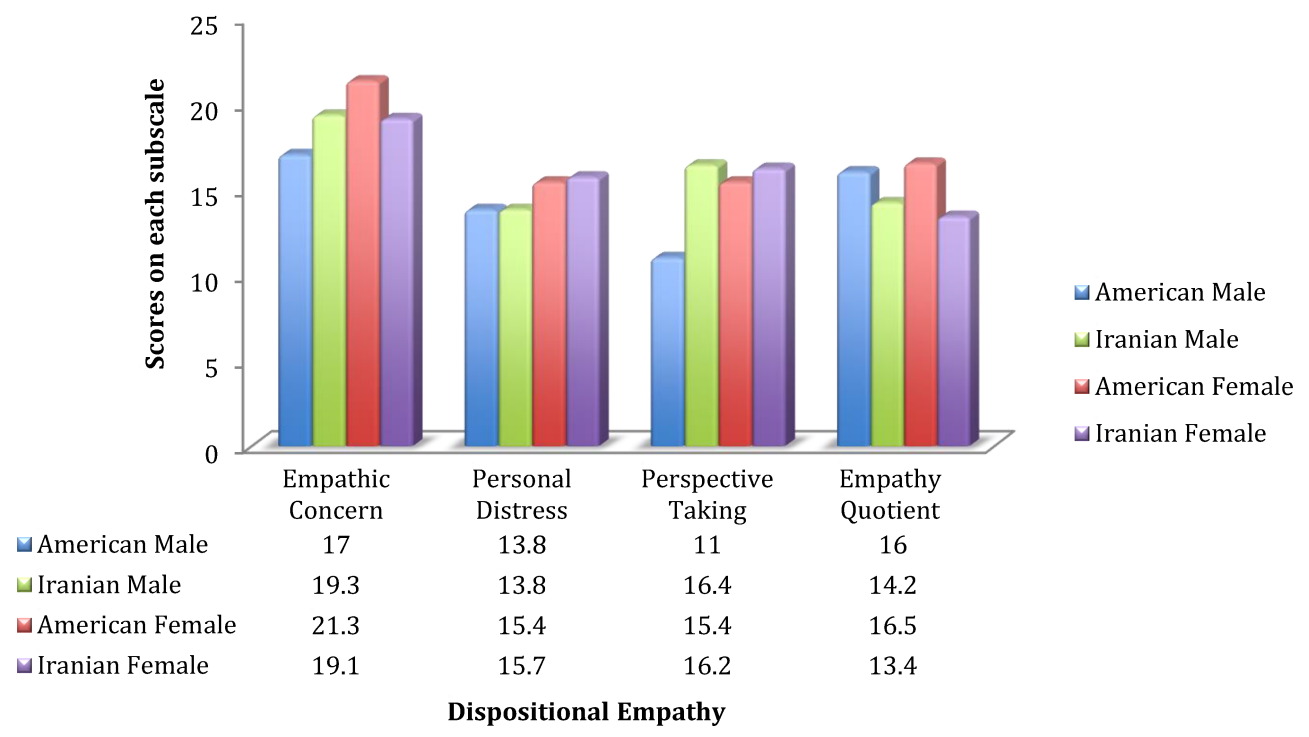


Cross-Cultural Approach to Empathy 